\title{
Dictynna
}

Dictynna

Revue de poétique latine

14 | 2017

Varia

\section{Carmenta ed Egeria : due ispiratrici silenziose nei Fasti di Ovidio}

Marco Fucecchi

\section{(2) OpenEdition}

Journals

Edizione digitale

URL: http://journals.openedition.org/dictynna/1432

DOI: $10.4000 /$ dictynna. 1432

ISSN: 1765-3142

\section{Notizia bibliografica digitale}

Marco Fucecchi, «Carmenta ed Egeria : due ispiratrici silenziose nei Fasti di Ovidio », Dictynna [En ligne], 14 | 2017, mis en ligne le 21 novembre 2017, consulté le 11 septembre 2020. URL : http:// journals.openedition.org/dictynna/1432; DOI : https://doi.org/10.4000/dictynna.1432

Questo documento è stato generato automaticamente il 11 settembre 2020.

\section{(c) (i) (9)}

Les contenus des la revue Dictynna sont mis à disposition selon les termes de la Licence Creative Commons Attribution - Pas d'Utilisation Commerciale - Pas de Modification 4.0 International. 


\title{
Carmenta ed Egeria : due ispiratrici silenziose nei Fasti di Ovidio
}

\author{
Marco Fucecchi
}

\section{NOTE DELL'AUTORE}

Desidero qui ringraziare i direttori della rivista e i due anonimi referees : le loro osservazioni mi hanno aiutato a migliorare alcuni aspetti di questo lavoro, che dedico all'amico Alain Deremetz. Degli errori rimasti rimango ovviamente l'unico responsabile.

\section{a) Introduzione}

1 Il poema elegiaco sul calendario romano è un'opera polifonica, dove il succedersi di molte voci diverse serve a drammatizzare (e a tematizzare) l'operazione di raccolta di notizie erudite e la ricerca documentaria relativa a nomi, rituali e festività. La voce del narratore principale interagisce con quelle di numerosi informatori divini, da lui espressamente evocati o che al contrario gli si presentano spontaneamente in aiuto (come Giano, nel libro I, e Giunone, nel libro VI), quasi ansiosi di sottomettersi ad un'intervista.

2 Questo genere di situazione, che ricorre più volte nel corso del poema, si basa su uno schema piuttosto consolidato, malgrado la presenza di alcune varianti ${ }^{1}$. In virtù di questo espediente, il poeta-eziologo 'entra' nel testo e mette in scena un dialogo tra sé e altri personaggi, rinunciando al presupposto (e al privilegio) dell'onniscienza per lasciarsi conferire una sorta di (auto-)legittimazione da un'autorità 'esterna'. Agli occhi del lettore, egli finisce così per assomigliare a un allievo piuttosto che al maestro dell'ars amatoria, depositario di una conoscenza accumulata con l'esperienza personale².

3 Davanti alla pluralità di opzioni possibili, il narratore effettua talvolta una scelta in favore di una causa piuttosto che di un'altra, oppure più spesso allinea - senza 
necessariamente classificarle in ordine di importanza - spiegazioni diverse di una festa o di un'usanza, come accade nei manuali di antiquaria, e come fanno del resto anche alcuni dei suoi stessi informatori divini (Giano è il primo esempio di questa tipologia).

4 D'altra parte, la moltiplicazione delle fonti di informazione e dei 'co-autori' non rappresenta un fenomeno unicamente costruttivo, né offre di per sé maggiori garanzie di obiettività. Soprattutto perché la collaborazione prestata dagli informatori non è propriamente gratuita, né disinteressata: questi interlocutori divini non dissimulano quasi mai la motivazione personale e la volontà di autopromozione che li spingono a collaborare. Nei Fasti un simile fenomeno può generare delle tensioni e alimentare perfino sospetti sull'affidabilità degli stessi informatori: sospetti che finiscono per indebolire anche l'autorevolezza della voce principale, che si trova a disposizione una varietà di eziologie concorrenti ed è chiamata a doversi pronunciare sulla maggiore $o$ minore qualità delle sue fonti ${ }^{3}$. $\mathrm{E}$, spesso, tale compito è reso ancor più difficile dalla valutazione 'diplomatica' dei rischi che una singola scelta potrebbe comportare, qualora non dovesse corrispondere alle attese di potenti interlocutori divini.

5 Così, non sono rari i casi in cui il poeta-eziologo preferisce sospendere il giudizio, evitando di prendere una posizione che potrebbe rivelarsi pericolosa per la sua stessa incolumità. Se la paura di scegliere lo blocca perfino quando si trova al cospetto di tre Muse (Polimnia, Urania e Calliope), che discutono con ammirevole fair-play sull'origine del nome del mese di maggio (fast. 5,1-110) ${ }^{4}$, non c'è proprio da meravigliarsi che egli non se la senta di dirimere la querelle, invero abbastanza tesa, che ha luogo all'inizio del mese di giugno, quando Giunone fa appello a tutta la sua esperienza di veterana per difendersi dall'attacco di avversarie agguerrite ed emergenti, come Ebe-Iuventas e Concordia (fast. 6,1-100). Nella circostanza, la sospensione del giudizio, da parte del poeta-eziologo, assume il carattere di un prudente gesto diplomatico, di una dimostrazione di savoir-faire cortigiano; ovvero, diventa un espediente scenico per drammatizzare la rinuncia ad esercitare prerogative proprie di un'autorità che non ammette discussioni.

\section{b) Voci che parlano e 'voci silenziose'}

6 Non intendo, però, soffermarmi in questa sede sui dialoghi che vedono protagonista il poeta ed uno o più informatori: dialoghi le cui modalità di attuazione sono già state, peraltro, ampiamente classificate. Mi occuperò, invece, di alcune figure divine femminili, a cui il testo ovidiano attribuisce la funzione di ispirare, oltre al poetaeziologo, anche altri personaggi. Si tratta di Carmenta o Carmentis - profetessa e madre dell'eroe arcade Evandro e, successivamente, assunta nel pantheon romano come divinità protettrice delle nascite -, e di Egeria, la ninfa consigliera e sposa del re Numa Pompilio.

7 Queste suggeritrici divine fanno (forse) parte del novero delle Camenae, le divinità italiche della poesia e dell'ispirazione, o comunque vengono ad esse tradizionalmente associate a vario titolo ${ }^{5}$. Entrambe, Carmenta ed Egeria, giocano un ruolo molto importante nei racconti in cui pure compaiono soltanto come personaggi complementari: un ruolo che risente in particolare della loro funzione primaria di consigliere e 'ammonitrici'b. In qualità di personaggi, esse prendono la parola soltanto per rivolgersi ai loro protetti, che sono, a loro volta, i protagonisti dei racconti medesimi: figure a cui esse sono strettamente legate, talvolta anche da rapporti di 
parentela, e ai quali danno dei consigli o suggeriscono modi di agire e di comportarsi. Da parte sua, il narratore può solamente invocarne l'aiuto, chiedere loro di rivelargli delle storie o suggerirgli delle spiegazioni. Le due divinità rispondono effettivamente a queste sue richieste, ma senza intervenire sulla scena di persona e senza rivolgerglisi direttamente come interlocutrici: in questo senso possiamo considerarle come delle vere e proprie figure dell'ispirazione poetica.

8 Ad ogni modo, la loro funzione va ben oltre l'ambito delimitato dalla finzione narrativa e finisce per esercitare una sorta di controllo, indiretto e occulto, sulle parole del narratore. La voce di quest'ultimo è punteggiata di numerose inflessioni celebrative che possono essere ricondotte in buona parte al bisogno di autopromozione che caratterizza queste ispiratrici silenziose. Non siamo molto lontani da quel modello di 'narrazione sotto tutela' che Alessandro Barchiesi ha individuato come un tratto distintivo di un certo numero di episodi dei Fasti: una nozione che traduce in evidenza testuale il complicato gioco dei rapporti di forza tra i diversi 'poteri' del racconto, e che volta per volta interviene a giustificare le modalità di costruzione del racconto medesimo ${ }^{7}$.

9 Tutto questo, comunque, si espone ad un'obiezione motivata: se Egeria e Carmenta comunicano esclusivamente con personaggi come loro, e non si rivolgono mai direttamente al poeta-eziologo (il cui statuto, in quanto personaggio, è del tutto particolare), com'è possibile considerare i suggerimenti impliciti che esse gli forniscono alla stregua di manifestazioni di 'voce interna' in senso proprio? In questo caso, non assistiamo infatti ad alcun dialogo tra il poeta-eziologo e un interlocutore; e, di conseguenza, la voce del narratore principale - pur condizionata dall'influsso della singola ispiratrice invocata nella circostanza - è la sola che riusciamo a udire. L'analisi delle modalità di interazione fra istanze differenti, all'interno di un contesto che si mantiene strettamente diegetico, ci porta inevitabilmente a sconfinare in un altro ambito di competenza: quello che concerne il punto di vista narrativo e la focalizzazione del racconto. Le ispiratrici silenziose agiscono in maniera subliminale: influenzano il modo del racconto; non prendono la parola per 'informare' il narratore, ma gli danno suggerimenti che finiscono per 'informare' la narrazione.

10 Nondimeno, questo non è che un primo aspetto della questione. Quando, infatti, le due divinità ispiratrici prendono finalmente la parola per aiutare i loro confidenti abituali, le loro voci sembrano veicolare allusioni indirette alla situazione di un personaggio che rimane esterno al poema: l'autore reale o empirico, ovvero colui del quale il poetaeziologo è una delle possibili proiezioni interne al testo (la più convenzionale, quantomeno). Per quanto risultino del tutto aderenti alle esigenze dei rispettivi contesti, i discorsi di Carmenta ed Egeria toccano anche temi che possono rimandare indirettamente alla storia personale di Ovidio in esilio, o almeno alla rappresentazione che il poeta dà di se stesso come esule: per es. il tema della necessità di sopportare le conseguenze della collera divina in attesa di tempi migliori, o quello della possibilità concreta di un'espiazione e della speranza di ottenere il perdono. Mentre forniscono delle risposte consolatrici, ovvero suggerimenti 'strategici', ai rispettivi confidenti, le due divinità ispiratrici utilizzano - senza di fatto violare le convenzioni narrative immagini e motivi resi celebri dall'elegia 'triste'.

11 Si ripete così - ma a ruoli invertiti - un procedimento analogo (o quasi) a quello che fa risuonare all'interno della voce 'unitaria' del narratore la traccia di altre voci, voci 'silenziose'. Questa volta sono le voci dei personaggi stessi, Carmenta ed Egeria, a 
manifestare tracce di un punto di vista superiore, che per un istante getta luce su una situazione extradiegetica, quella di Ovidio esule: un fenomeno che non definirei propriamente come focalizzazione alternativa, o 'deviante's, ma piuttosto 'concorrente'. Uno scambio di prerogative, dunque, che accanto alla voce del narratore - 'controllata' da divinità che non hanno bisogno di prendere direttamente la parola pone la voce di queste stesse figure ispiratrici, che (indirettamente) si mostrano al tempo stesso solidali verso l'autore empirico', secondo la rappresentazione poetica che di lui offrono Tristia ed Epistulae ex Ponto.

\section{c) Carmenta}

Ovidio accetta la relazione etimologica tra il nome di Carmenta e carmen presupposta da Virgilio (fast. 1,467 s. ipsa mone, quae nomen habes a carmine ductum, / propositoque fave ne tuus erret honor, 'istruiscimi tu, che derivi il tuo nome dal canto (carmen), e sii favorevole a quest'impresa, affinché nell'onorarti io non incorra in un errore'). Tuttavia, a differenza di quest'ultimo, egli presenta fin da subito Carmenta (o Carmentis) come una dea (fast. 1,462 Arcadiae ... deae). Nell'Eneide, invece, la madre di Evandro era ricordata come una ninfa dal figlio stesso (8,335 s. matrisque ... tremenda / Carmentis Nymphae monita, 'i terrificanti ammonimenti di mia madre, la ninfa Carmenta') e dal narratore extradiegetico (338 s. Carmentalem ... portam / ... Nymphae priscum Carmentis honorem, 'la porta Carmentale, antico tributo alla ninfa Carmenta') 9 .

13 Nei Fasti Carmenta è invocata come ispiratrice in occasione del primo giorno della sua festa, i Carmentalia dell'11 gennaio ${ }^{10}$. Dopo aver guidato la navigazione del figlio Evandro, esiliato insieme a lei dall'Arcadia, Carmenta - che ormai è divenuta una dea romana perfettamente integrata, un vero modello di migrazione fortunata - sarà guida affidabile anche della navigazione poetica, rispondendo efficacemente alla richiesta iniziale del narratore (v. 466 deriget in medio quis mea vela freto?, 'chi governerà le mie vele in mezzo alle onde?') ${ }^{11}$. Richiamando a questo punto un'immagine che caratterizzava già il primo proemio, dove il poeta rivolgeva a Germanico la richiesta di guidare la sua impresa letteraria (fast. 1,4 hoc opus et timidae derige navis iter, 'guida quest'opera e la rotta della mia nave timorosa'), Ovidio mostra di attribuire a Carmenta notevole importanza anche come figura dell'ispirazione poetica ${ }^{12}$.

14 Le qualità profetiche di Carmenta costituiscono, invece, l'argomento principale di un racconto-cornice, in cui il narratore prende la parola all'inizio $(1,465-78)$ e alla fine, quando - dopo aver raccontato la vittoria di Ercole su Caco (aition dell'ara Maxima: 1,543-82) - chiude 'ad anello' con un ultimo omaggio dedicato proprio alla madre di Evandro (1,583-6). La parte centrale (1,479-542) è, al contrario, letteralmente dominata dalla voce della stessa Carmenta. In due lunghe sequenze di discorso diretto, ella dapprima incoraggia il figlio ad affrontare l'esilio con fermezza e spirito di sacrificio (479-96), e quindi - preda di un furore che costringe Evandro a trattenerne l'impeto ${ }^{13}$ profetizza l'arrivo di Enea e la nascita di Roma, arrivando fino all'attualità, con la celebrazione di Livia e Tiberio, madre e figlio come lei ed Evandro (509-36).

15 Queste due rheseis sono, abbiamo detto, incorniciate e collegate tra loro da segmenti celebrativi che elogiano la profetessa e, insieme, la 'navigatrice' affidabile (contrariamente ad Anchise, Carmenta indica sempre la rotta giusta!) e fortunata (540 felix, exilium cui locus ille fuit!, 'felice colui al quale è tocccato questo come luogo di esilio'). Il narratore ha chiesto, ed evidentemente ottenuto, l'aiuto particolare di 
Carmenta come veggente ed ispiratrice del canto poetico ${ }^{14}$. Egli ha proposto un accordo alla dea, che ha tutto l'interesse a vedere valorizzata la propria immagine $(1,468$ propositoque fave, ne tuus erret honor, 'sii favorevole a quest'impresa, affinché nell'onorarti io non incorra in un errore'). Pertanto, non c'è da stupirsi se la narrazione seguente enfatizza soprattutto la nobiltà della madre, la cui reputazione giunge ad oscurare la pur illustre (ma, almeno qui, non meglio specificata) origine paterna di Evandro (1,471 s. hinc fuit Evander, qui, quamquam clarus utroque, / nobilior sacrae sanguine matris erat, 'di qui (= l'Arcadia) era Evandro, che, sebbene illustre per entrambi i natali, era più nobile per via della discendenza materna') ${ }^{15}$.

16 Secondo la tradizione ufficiale riferita da Dionigi di Alicarnasso - e alla quale alludono probabilmente anche Virgilio e lo stesso Livio -, Carmenta avrebbe avuto Evandro nientemeno che da Hermes, il dio della scrittura ${ }^{16}$. Ovidio potrebbe, dunque, presupporre un'altra tradizione: in effetti sembra difficile che Mercurio (per quanto $e$ silentio) sia considerato inferiore a Carmenta! $\mathrm{E}$, del resto, qualche labile traccia di versioni antagonistiche esiste: in alcune note di Servio, la madre di Evandro - sorella di Elena e Clitemnestra - si chiama Timandra ed è sposata con un certo Echemus; oppure, ancora, è una figlia di Mercurio e il suo nome è Nicostrata ${ }^{17}$. Rimane, in alternativa, la possibilità di pensare che - proprio in omaggio all'ispirazione' ricevuta - il narratore abbia voluto esaltare la figura materna, mettendo diplomaticamente la sordina alla nobiltà olimpica del padre di Evandro.

17 L'intenzione di sottolineare le doti profetiche della dea festeggiata in gennaio è confermata subito dopo. Disgraziatamente, la voce di Carmenta si è rivelata fin troppo veritiera, quando ha predetto avvenimenti dolorosi, come l'esilio per sé e per suo figlio (1,473-8 quae, simul aetherios animo conceperat ignes, / ore dabat vero carmina plena dei. / dixerat haec nato motus instare sibique, / multaque praeterea tempore nacta fidem. / nam iuvenis nimium vera cum matre fugatus / deserit Arcadiam Parrhasiumque larem, "non appena aveva concepito in cuor suo l'ardore dell'ispirazione celeste, costei pronunciava con bocca veritiera oracoli pregni del nume divino. Sempre lei aveva annunciato i pericoli incombenti sul figlio e su se stessa, e molti altri avvenimenti, che si sarebbero avverati col tempo. E in effetti, scacciato assieme alla madre fin troppo veridica, il giovane abbandona l'Arcadia e la sua dimora parrasia'). Ma veritiera la profetessa è stata anche quando ha indicato a Evandro la rotta verso un futuro radioso (1,497 ss., in part. 499-500 iamque ratem doctae monitu Carmentis in amnem / egerat [scil. Evander], 'egli (Evandro), seguendo il consiglio di Carmenta, edotta sui fati, aveva già fatto entrare la nave nella foce del fiume') $)^{18}$.

Il narratore mostra, così, di meritare la fiducia che gli è stata accordata dall'ispiratrice divina. Dunque, ora non ci resta che cercare qualche indizio di 'gratitudine' nei suoi confronti all'interno delle parole di Carmenta. Partiamo, perciò, dal primo dei discorsi diretti della profetessa, la consolatio che funziona anche come un protrettico. La madre ridà coraggio al figlio, sgravandolo di ogni responsabilità: è stato il destino a imporgli di lasciare la patria; non è colpa sua se ha dovuto partire in esilio, ma della collera vendicativa (e imperscrutabile) di un dio (1,481-6 sic erat in fatis; nec te tua culpa fugavit, / sed deus: offenso pulsus es urbe deo. / non meriti poenam pateris, sed numinis iram: / est aliquid magnis crimen abesse malis. / conscia mens ut cuique sua est, ita concipit intra / pectora pro facto spemque metumque suo, 'stava scritto così nel destino; non sei tu la causa che ha portato al tuo esilio, ma un dio: tu sei stato bandito dalla città per via dell'ostilità di un nume: vale pur qualcosa l'essere innocenti, quando si è afflitti da grandi disgrazie! 
Ciascuno, secondo la testimonianza della propria coscienza, prova in cuor suo speranza o timore in relazione a ciò che ha compiuto'). In questo passo il poeta delega al personaggio parlante una possibile, indiretta, allusione - dal passato remoto - alla situazione dell'autore empirico', o - se si preferisce - all'immagine testuale che di lui ci ha consegnato l'elegia triste.

In effetti, anche sull'esilio di Evandro circolano tradizioni diverse ${ }^{19}$, ma nessun'altra testimonianza - a parte questo passo dei Fasti - ne riconduce la ragione unicamente al motivo della 'collera di un dio': e, per di più, attraverso una formula che - oltre alle prime parole rivolte dal vate marino Proteo ad Aristeo in Virgilio (georg. 4,453 non te nullius exercent numinis irae, 'è l'ira di qualche nume che ti perseguita') - richiama alla memoria un tema ricorrente nei Tristia e nelle Epistulae ex Ponto ${ }^{20}$.

La presenza di possibili richiami indiretti all'esilio di Ovidio in questo passo è stata notata da tempo: circa venticinque anni fa Elaine Fantham ha dedicato un'analisi sistematica a questi indizi di allusione riflessiva ${ }^{21}$. Più di recente, Steven Green, commentatore del libro I dei Fasti (2004), ha mosso l'obiezione, secondo cui Ovidio nell'ambito dell'elegia triste - non si permette mai un genere di denuncia così esplicito $^{22}$. È pur vero, sostiene Green, che il poeta esule tende ad attenuare la gravità della propria culpa (tr. 1,2,98), e dice che si era trattato piuttosto di un error (tr. 1,2,99; 3,37 etc. 4,1,23 s.), o che - se proprio di colpa si doveva parlare - essa era stata involontaria, come quella di Atteone (tr. 2,103 s.). Ma egli non si spinge mai al punto di negare che la collera del dio era giustificata, così come lo era la punizione che lui, il poeta, doveva scontare (tr. 3,1,51 s.): anzi, talvolta, ammette perfino di aver commesso un torto grave (Pont. 2,2,15) e che la relativa pena, per quanto giunta in notevole ritardo (tr. 2,545 s., in relazione all'ars), era stata inferiore a quanto avrebbe meritato di ricevere (Pont. 1,2,96 e 3,3,76).

21 Io non credo, tuttavia, che questo diminuisca necessariamente la portata dell'allusione contenuta nel discorso di Carmenta: una divinità che conosceva la sofferenza dell'esilio e a cui il narratore-eziologo affida il compito di perorare - indirettamente - anche la causa dell'autore'. Come già in precedenza, anche qui sarebbe forse inutile ipotizzare una variante altrimenti sconosciuta del mito: il motivo della punizione inflitta ad Evandro da un dio sarà, piuttosto, da considerare alla stregua di un'integrazione e di un'allusione nascosta, ovvero come un gesto letterario dissimulato. È un po' come se Carmenta rendesse al poeta il favore che da lui ha ricevuto in precedenza: il suo statuto narrativo privilegiato le permette di affermare ciò che ad altri, e in particolare a colui che scrive dall'esilio, non è né permesso, né consigliabile, esprimere verbalmente.

L'efficacia della consolatio e il suo possibile significato extradiegetico sono corroborati dalla verità (questa sì, incontestabile) della profezia che la segue. Carmenta saluta la terra che darà al cielo nuove divinità: Enea, Romolo, Cesare, Augusto e Livia (v. 510 tuque, novos caelo terra datura deos); la terra che ospiterà anche la città-mondo del futuro, o meglio del presente (515 s. fallor, an hi fient ingentia moenia colles / iuraque ab hac terra cetera terra petet?, 'forse mi sbaglio, o queste colline diventeranno mura possenti e tutta la terra domanderà leggi e diritto a questa terra?'); la sede della dinastia imperiale (527-36). Ora è la dea stessa a tematizzare la nozione della propria veridicità e affidabilità (518 quis tantum fati credat habere locum?, 'chi potrebbe credere che questo luogo abbia davanti un futuro così grande?'): e la realtà è sotto gli occhi di tutti per conferire a simili parole una legittimazione 'a posteriori'. 
riferisce l'episodio dei carpenta. Nella circostanza, la dea ispira 'silenziosamente' la riconciliazione tra matrone e senatori, che garantirà a Roma una discendenza (1,617-28). Come al solito, la selezione del materiale e la distribuzione dei ruoli sono attente e calibrate: la voce di Evandro non si ode più per tutto il resto dell'episodio e la rievocazione dell'impresa di Ercole (invero un po' lontana dall'anniversario dell'ara Maxima, che cade il 12 agosto) è affidata al narratore che conferisce tra l'altro all'eroe semidivino un carattere sacerdotale (e augusteo): forse un ulteriore omaggio indiretto a Carmenta e, al tempo stesso, un messaggio istruttivo per Evandro e per il principe del futuro $^{25}$. La narrazione del vates operose dierum ha ricominciato a fluire in modo composto e dignitoso: la breve inflessione personale, che aveva lasciato una traccia nella voce di Carmenta e in un fugace commento 'autoriale' sul (migliore, quasi invidiabile) destino dell'esule Evandro, non è ormai che una semplice eco del passato.

\section{d) Egeria}

La figura di Egeria manifesta diversi tratti in comune con Carmenta: anche lei può essere associata alle Camene; anche lei (come Carmenta rispetto a Giano) fa da pendant contrastivo al ruolo di Marte, il dio patrono del mese (marzo) in cui è festeggiata, nel senso che - contrariamente a quest'ultimo ${ }^{26}$ - anche lei ispira il poeta con discrezione e, soprattutto, rimanendo di fatto in silenzio, senza apparirgli davanti né accordargli alcuna intervista.

Tuttavia il contrasto fra Egeria e Marte sembra assai più netto di quello tra Carmenta $\mathrm{e}$ Giano, e riflette in buona parte la diversa natura dei loro rispettivi protégés: Numa, 
esempio - come Evandro - di un potere pacifico, e Romolo, bellicoso figlio di Marte e fondatore di Roma. Come ha giustamente osservato Elena $\mathrm{Merli}^{27}$, Egeria viene invocata dal narratore allo stesso modo di Carmenta (3,259 ss. quis mihi nunc dicet...? nympha, mone etc. 1,465 ss. unde petam causas ... ? ipsa, mone), e interviene a sostituire Marte come informatore proprio quando il tema proposto sembra riguardare il dio più da vicino, ovvero al momento di trattare l'origine degli ancilia, gli scudi agitati dai sacerdoti Salii: una storia che esalta la pietas e, al tempo stesso, la sagacia dialettica e diplomatica di Numa (3,277 ss. $)^{28}$.

Questo si verifica, d'altra parte, dopo che il dio della guerra ha già raccontato (3,173-252) il ratto delle Sabine (un escamotage da lui 'consigliato' personalmente al figlio: 198), nonché illustrato una serie di ipotesi, abbastanza disparate, sulla propria relazione con la festa dei Matronalia ${ }^{29}$. Alla fine del suo lungo discorso diretto, facendo leva sulla sua dubbia (o, quantomeno, discutibile) nascita da Giunone ${ }^{30}$, Marte pretende poi di essere addirittura il destinatario di un culto particolare in qualità di figlio della dea protettrice delle matres (3,251 mater amat nuptas: matrum me turba frequentat, 'mia madre ama le giovani spose: le madri accorrono in massa a celebrare la mia festa'): tutto questo mostra, comunque, la difficoltà di Marte nel costruire una plausibile immagine auto-elogiativa e nell'accreditarsi come dio pacifico.

29 Egeria entra, dunque, in azione subito dopo che il discorso è andato a finire sulle matrone romane, invitate a ricercare il favore di un'altra divinità protettrice del parto (dopo Carmenta), cioè (Giunone) Lucina (3,253-6). È a questo punto che il narratore invoca l'aiuto della sposa di Numa, la ninfa che gli ispira il racconto degli ancilia e che sarà lei stessa attrice effettiva (benché secondaria) di questa storia (3,259 ss. quis mihi nunc dicet quare caelestia Martis / arma ferant Salii Mamuriumque canant? / nympha, mone, nemori stagnoque operata Dianae; / nympha, Numae coniunx, ad tua facta veni, "chi mi dirà adesso perché i Salii portino le armi di Marte cadute dal cielo, e perché intonino canti in onore di Mamurio? o ninfa, che sei al servizio di Diana nel suo bosco e presso il suo lago, insegnamelo tu; o ninfa, sposa di Numa, vieni e aiutami a celebrare le tue gesta') ${ }^{31}$.

La presentazione ufficiale di Egeria $(3,261-76)$, incorniciata dalla sua definizione come Numae coniunx (262 e 276), contiene un'immagine simbolica dell'ispirazione poetica che da lei promana: il poeta raffigura se stesso nell'atto di bere a piccoli sorsi (274 exiguis haustibus) da un ruscello alimentato dalla sorgente di Egeria (3,275 Egeria est quae praebet aquas, dea grata Camenis, 'è lei, Egeria, che fornisce l'acqua, dea cara alle Camene'). Attraverso l'acqua della sorgente ${ }^{32}$, la ninfa consigliera - senza prendere direttamente la parola, proprio come Carmenta - 'suggerisce' al narratore il racconto dell'impresa di Numa, un'impresa in cui ha giocato un ruolo importante anche lei. In quanto personaggi di racconto, dunque, Egeria e Carmenta sono rappresentate mentre rivolgono le loro parole ad altri personaggi (Numa, Evandro) e, al tempo stesso, ispirano il narratore.

Il passaggio di consegne tra Romolo e Numa viene, così, marcato anche dal concomitante avvicendamento fra le rispettive divinità ispiratrici dei due sovrani. A Marte - il dio che aveva alimentato il furore combattivo di Romolo, suggerendogli tra l'altro l'escamotage del ratto delle Sabine per popolare la sua città - succede Egeria, consigliera di pace. Lo scudo che il re sabino riceve da Giove - grazie ai saggi consigli della sposa divina - sancisce la legittimazione ufficiale del suo potere: in seguito egli governerà la città come un sovrano benevolo e pacifico. D'altra parte, almeno su un piano culturale, questa 'successione' si è già realizzata all'interno del libro III dei Fasti. 
Dopo la lunga digressione sulla scelta di Romolo di dedicare al padre Marte il primo mese dell'anno (3,71-150), il narratore spiega infatti che fu Numa il primo a rendersi conto che al calendario romuleo mancavano due mesi (3,151 s. primus, oliviferis Romam deductus ab arvis, / Pompilius menses sensit abesse duos, 'il primo ad accorgersi che mancavano due mesi fu Numa Pompilio, giunto a Roma da campi fecondi di ulivi'). Quindi, egli continua dicendo che un suggerimento tanto sapiente poteva essere giunto al re in due modi: dal filosofo Pitagora - che, secondo una improbabile tradizione, era stato maestro di Numa a Taranto - o, appunto, dalla sua consigliera abituale, Egeria $(3,154 \ldots \text {... Egeria sive monente sua })^{33}$. L'esaltazione delle qualità e delle imprese di Numa è, senza dubbio, l'argomento principale di questa parte del libro III: il re sabino occupa quasi da solo la scena, senza dover condividere questo onore, come capita invece ad Evandro alla fine del libro I, dove si tratta soprattutto di festeggiare Carmenta. Nondimeno, la figura di Egeria - a cui il narratore ha domandato l'ispirazione - lascia alcune tracce significative, e la sua posizione all'interno del programma celebrativo che caratterizza l'episodio non appare affatto inessenziale, malgrado le sia assegnato un ruolo drammatico da semplice comprimaria.

Inoltre sembra verificarsi a questo punto un fenomeno di 'reciprocità' non molto diverso da quello osservato in occasione dei Carmentalia dell'11 gennaio. All'inizio della storia degli ancilia, Egeria prende la parola per incoraggiare il marito a tentare di placare la tempesta di fulmini inviata da Giove. A tal fine, gli consiglia di rivolgersi a chi potrà aiutarlo nell'impresa di avvicinare il dio più grande di tutti (3,289-94: cui dea 'ne nimium terrere: piabile fulmen / est' ait 'et saevi flectitur ira Iovis. / sed poterunt ritum Picus Faunusque piandi / tradere, Romani numen utrumque soli. / nec sine vi tradent: adhibe tu vincula captis'; / atque ita qua possint edidit arte capi, 'la dea gli dice: "non lasciarti intimorire troppo: il fulmine si può scongiurare e la collera di Giove, quando si scatena feroce, può essere piegata. Ma il rito di espiazione potranno insegnartelo Pico e Fauno, che sono entrambi divinità indigene del territorio di Roma. Ma non te lo insegneranno senza essere costretti con la forza: devi esser tu a catturarli con i lacci"; e gli rivelò in che modo potevano essere catturati').

Questa breve sequenza di discorso diretto, che contiene un'esortazione e alcune generiche indicazioni operative, non è affatto paragonabile alle due lunghe rheseis pronunciate da Carmenta, che dimostrava nella circostanza il proprio talento oratorio (la consolazione parenetica) e divinatorio (la profezia sull'avvento di Roma). Malgrado ciò, essa contiene alcuni elementi interessanti ai nostri fini. All'inizio la dea rincuora il marito, rassicurandolo sull'effettiva possibilità di placare l'ira terribile del sovrano degli dèi (3,289 s. ... ne nimium terrere; piabile fulmen / est etc.). Quindi - come aveva fatto Cirene, madre e consigliera di Aristeo nel libro IV delle Georgiche virgiliane - anche Egeria consiglia a Numa di ricercare la collaborazione di due preziosi intermediari, Pico e Fauno, divinità indigene che sanno come attirare Giove e potranno, perciò, indurlo a scendere sulla terra e ascoltare la richiesta di Numa.

Qui le parole di Egeria, che di norma è consigliera pacifica, stimolano il pio e religiosissimo Numa a lanciare addirittura una sfida al sovrano divino: una sfida, peraltro, giusta e legittima, con cui il re sabino non intende certo ingaggiare un'irriverente competizione, quanto piuttosto ricondurre Giove a più miti consigli $\mathrm{e}$ ottenere un beneficio per il suo popolo. L'incoraggiamento iniziale di Egeria fa venire in mente una riflessione del poeta di Tristia 2 riguardo alla possibilità di ricevere il perdono ed essere finalmente richiamato a Roma. Dopo aver sviluppato il tema della 
collera giustificata, e perfino troppo 'controllata' del princeps-dio (che a ragione lo ha allontanato dal caput mundi), la voce del poeta manifesta un'audace speranza: una speranza, certo, assai poco ragionevole, ma alimentata dalla natura benigna del dio stesso; il sogno di ritornare a vivere dopo che la vita stessa è stata annientata dalla violenza della folgore: Ov. tr. 2,141 ss. sed solet interdum fieri placabile numen; / nube solet pulsa candidus ire dies. / vidi ego pampineis oneratam vitibus ulmum, / quae fuerat saevo fulmine tacta Iovis ('ma talvolta il nume divino può anche lasciarsi placare; di solito, quando le nuvole sono state dissipate, il giorno torna a risplendere. Io stesso ho visto carico di pampini e di uve un olmo, dopo che era stato colpito dalla terribile folgore di Giove'). La possibilità (e l'auspicio) di scongiurare la folgore di Giove, concreta manifestazione dell'ira divina, viene espressa in modo analogo nei due contesti (fast. 3,290 piabile fulmen trist. 2,142 placabile numen), e ciò rappresenta un altro elemento ricorrente nell'immaginario dell'elegia triste ${ }^{34}$.

La stessa formula richiama abbastanza da vicino le ultime parole della consolatio di Carmenta ad Evandro (fast. 1,495 s. nec fera tempestas toto tamen horret in anno: / et tibi, crede mihi, tempora veris erunt, "per quanto aspra, una tempesta non infuria per un anno intero: e anche per te, credimi, verrà la stagione di primavera'), che si possono confrontare anche con tr. 5,8,31 s. si numeres anno soles et nubila toto, / invenies nitidum saepius isse diem ('se, nel corso di un anno, conti i giorni di sole e quelli nuvolosi, alla fine vedrai che ha prevalso il sereno'). Questa fiducia si rivela, così, una sorta di ideale trait d'union fra le parole che le due ispiratrici silenziose del poeta rivolgono ai loro beniamini. Come in occasione della profezia di Carmenta al figlio, anche le parole con cui Egeria incoraggia Numa ad affrontare Giove dovrebbero - se ricondotte alla situazione dell'autore' colpito dall'ira divina - suonare non tanto come una consolazione 'ex ante', quanto piuttosto come un auspicio promettente, che riecheggia dal passato lontanissimo.

Ancora una volta la voce di un personaggio esprime un'istanza condivisa e appare influenzata da un punto di vista sovraordinato, da una focalizzazione superiore. Il messaggio, in questo caso, non è soltanto la speranza di una riabilitazione e di un ritorno, ma anche - più in generale - una testimonianza di fede nel potere rigeneratore della poesia e dell'arte. Gli dèi minori Pico e Fauno (anch'essi abituati ad abbeverarsi ad una fonte sacra, 'gestita' da Egeria: 3,297-9), ma soprattutto l'artigiano Mamurio, che fabbrica le copie dell'ancile disceso dal cielo, sono personaggi che mettono la loro 'arte' al servizio del potere, contribuendo a consolidare il buon governo di un sovrano virtuoso, che viene indicato come esempio: una funzione collaborativa che non sembra poi troppo divergente da quella che propone il vates operose dierum. Se la voce di quest'ultimo opera al fine di orientare la celebrazione poetica verso una direzione precisa, è attraverso le parole di Carmenta ed Egeria - le 'ispiratrici' che traggono lustro e prestigio dal discorso del poeta - che noi lettori possiamo figurarci l'influenza di uno sguardo ulteriore sul racconto, che del racconto stesso amplifica e approfondisce il senso.

\section{e) Un breve bilancio}

37 All'interno del poema elegiaco sul calendario, ricco di presenze e di voci che dialogano con il narratore, istruendolo sull'origine di festività, di nomi e di rituali, ho ritenuto di poter isolare un piccolo sottoinsieme di ispiratrici/informatrici, di cui il poeta-eziologo 
invoca l'aiuto, ma che non gli appaiono mai davanti di persona, né mai gli si rivolgono direttamente usando la propria voce. Secondo la loro natura di mediatrici tra mondo degli uomini e mondo degli dèi - che ha indotto comunemente a identificarle (o almeno ad associarle) con le italiche Camenae, sorgenti divine dell'ispirazione - Carmenta ed Egeria si limitano, in effetti, a insinuare la loro voce nella mente del narratore, fedeli al ruolo che la tradizione assegna alle divinità della memoria e del canto poetico.

Tuttavia, esse condividono con le Muse dei due primi libri degli Aitia callimachei e con quelle che appaiono nella seconda parte degli stessi Fasti (Erato nel libro IV; Polimnia, Urania e Calliope all'inizio del libro V) anche lo statuto di attrici (e, talora, 'autrici') del racconto. Sia Carmenta che - sebbene in misura quantitativamente inferiore - Egeria recitano un ruolo importante nell'ambito dei rispettivi racconti di cui fanno parte. Ma la loro voce interagisce esclusivamente con altri personaggi come loro, e ciò avviene naturalmente - come per tutti i personaggi - in modo indiretto, per bocca del narratore interno.

Il fatto che, a differenza di altri informatori divini, Carmenta ed Egeria non siano protagoniste di un'epifania, né colgano l'occasione di una 'intervista' per fare autopromozione, non significa da parte loro la rinuncia ad esercitare comunque una forma di controllo sul racconto eziologico. Al contrario, la voce del poeta che esse ispirano assume inflessioni celebrative che - se non si possono definire come vere 'citazioni' - testimoniano quantomeno una sofisticata strategia di omaggio: dietro la voce del narratore si ha, cioè, l'impressione di poter scorgere talora il punto di vista di un'ispiratrice orgogliosa di sé. Quest'ultima può approfittare, così, della vantaggiosa circostanza di non doversi esporre direttamente allo scetticismo del lettore, come può capitare a certi (improvvisati) interlocutori divini cui non fa difetto l'autostima, ma che possono talora apparire un po' in imbarazzo ${ }^{35}$.

In cambio, il narratore principale non si lascia sfuggire le rispettive occasioni in cui le due ispiratrici divine prendono direttamente la parola come personaggi per fare in modo che esse gli restituiscano, per così dire, il favore. In concomitanza con i passaggi cruciali di veri e propri interventi oratori (le due lunghe rheseis di Carmenta) o di alcune brevi allocuzioni (l'incoraggiamento e il consiglio di Egeria a Numa), entrambe si fanno portavoce di istanze che riguardano da vicino lo stesso autore empirico, o che almeno sembrano adattarsi all'immagine che di lui viene costruita dal testo del poema sul calendario ${ }^{36}$. Una simile circostanza induce a ritenere che il tema dell'esilio - in quanto esperienza politica, psicologica e letteraria - possa aver giocato un ruolo importante anche nel programma dei Fasti, e magari aver informato di sé le fasi della loro revisione. Anche così, in una maniera più mediata e discreta, l'esule Ovidio sembra continuare a coltivare la speranza di ottenere il perdono del sovrano: ipse licet sperare vetes, sperabimus usque, 'benché tu stesso mi vieti di sperare, io continuerò a sperare' (tr. $2,145)$. 


\section{BIBLIOGRAFIA}

Ballentine 1904 :

Ballentine, Floyd G., 'Some Phases of the Cult of the Nymphs' in: HSCPh 15 (1904), 77-119;

Barchiesi 1991:

Barchiesi, Alessandro, 'Discordant Muses', in: PCPhS 37 (1991), 1-21;

Barchiesi 1994:

Barchiesi, Alessandro, Il poeta e il principe. Ovidio e il discorso augusteo, Roma-Bari 1994;

Battistella 2012:

Battistella, Chiara, 'La verità delle Muse: un dittico ovidiano (met. 5,250-664 e fast. 5,1-110)', in: Museum Helveticum 69 (2012), 96-102;

Buchheit 1993:

Buchheit, Vinzenz, 'Numa: Pythagoras in der Deutung Ovids', in: Hermes 121 (1993), 77-99;

Eden 1975:

Eden, Peter T., A Commentary on Virgil: Aeneid VIII, Leiden 1975;

Fantham 1992:

Fantham, Elaine R., 'The Role of Evander in Ovid's Fasti', in: Arethusa 25 (1992), 155-171;

Fowler 1990 :

Fowler, Don, 'Deviant Focalisations in Virgil's Aeneid', in: PCPhS 36 (1990), 42-63;

Fucecchi 2004:

Fucecchi, Marco, 'L'orgoglio dei meno grandi: autocoscienza, sagacia e abilità diplomatica di alcune divinità 'minori' dei Fasti', in: L. Landolfi (ed.), Nunc teritur nostris area maior equis. Riflessioni sull'intertestualità ovidiana - i fasti, Palermo 2004, 25-46;

Green 2004:

Green, Steven J., Ovid, Fasti 1. A Commentary, Leiden 2004;

Hardie 2007:

Hardie, Alex, 'Juno Hercules, and the Muses at Rome', in: AJPh 128 (2007), 551-592;

Hardie 2016:

Hardie, Alex, 'The Camenae in Cult, History and Song', in: Classical Antiquity 35 (2016) 45-85;

Hinds 1992:

Hinds, Stephen E., 'Arma in Ovid's Fasti. Part 2: Genre, Romulean Rome and Augustan Ideology', in: Arethusa 25 (1992), 113-153;

Horsfall 2000:

Horsfall, Nicholas, Virgil, Aeneid 7. A Commentary, Leiden-Boston-Köln 2000;

Labate 2010:

Labate, Mario, Passato remoto: età mitiche e identità augustea in Ovidio, Pisa/Roma 2010.

Merli 2000:

Merli, Elena, Arma canant alii: Materia epica e narrazione elegiaca nei fasti di Ovidio, Firenze 2000;

Merli 2013;

Merli, Elena, Dall'Elicona a Roma: acque ispiratrici e lima poetica nell'Ovidio dell'esilio e nella poesia flavia di omaggio, Berlin/Boston 2013; 
Miller 1982:

Miller, John F., 'Callimachus and the Augustan Aetiological Elegy', in: ANRW 2.30.1 (1982), 371-417;

Miller 1983:

Miller, John F., 'Ovid's Divine Interlocutors in the Fasti', in: C. Deroux (ed.), Studies in Latin

Literature and Roman History III, Bruxelles 1983, 156-192;

Newlands 1995:

Newlands, Carole E., Playing with Time: Ovid and the Fasti, Ithaca - NY 1995;

Pasco-Pranger 2006:

Pasco-Pranger, Molly, Founding the Year: Ovid's Fasti and the Poetics of the Roman Calendar, Leiden

2006;

Rutledge 1980:

Rutledge, Eleanor, 'Ovid's Informants in the Fasti', in: C. Deroux (ed.), Studies in Latin Literature and Roman History II, Bruxelles 1980, 322-331;

Schilling 1993:

Schilling, Robert (ed.), Ovide. Les Fastes. tome I-II. Texte établi, traduit et commenté par R.S., Paris 1993;

Stok 2004:

Stok, Fabio, 'Lo spettacolo degli ancilia', in: L. Landolfi (ed.), Nunc teritur nostris area maior equis. Riflessioni sull'intertestualità ovidiana - $i$ fasti, Palermo 2004, 69-79;

Tels-De Jong 1959:

Tels-De Jong, Léontine L., Sur quelques divinités romaines de la naissance et de la prophétie, Delft 1959;

Waszink 1956:

Waszink, Jan H., 'Camena', in: Classica et Medievalia 17 (1956), 139-148;

Wissowa 1902:

Wissowa, Georg, Religion und Kultus der Römer, München 1902.

\section{NOTE}

1. I primi studi che si occupano sistematicamente della questione sono Rutledge 1980; Miller 1982 e Miller 1983.

2. Newlands 1995,54.

3. Barchiesi 1994,169 s. e passim.

4. Barchiesi 1991; Labate 2010,210-14 e Battistella 2012 (con ulteriore, e più recente, bibliografia).

5. Wissowa 1902,180 ss. (Carmenta/Carmentis) e 200 s. (Egeria); Ballentine 1904,108 ss. ; Waszink 1956,139 ss. ; Tels-De Jong 1959, e ora soprattutto Hardie 2016 (che, peraltro, nega la possibilità di includerle fra le Camenae). Per la relazione tra Egeria e le Camenae, si possono vedere fast. 3,275; met. 15,482; Liv. 1,21,3; Mart. 6,47. Una tradizione alternativa, riferita da Dionigi di Alicarnasso (Ant.Rom. 2,60,5), faceva viceversa di Egeria una delle Muse. Per la natura divina di Carmenta, vedi anche più oltre, nel testo e alla n. 9 .

6. Per il collegamento tra questa funzione e l'origine delle Camenae da (Giunone) Moneta, dea della memoria e corrispettivo della greca Mnemosyne, cfr. Hardie 2007,556 s.

7. Barchiesi 1994,190 s.

8. Fowler 1990.

9. Sulla questione, Servio e Servio Danielino si dividono: il primo ritiene che nympha sia un epiteto poetico (haec autem non vere nympha fuit, sed vaticinatrix); il secondo, invece, include 
Carmenta tra le sex feminas ... quae nymphae vocarentur in Arcadia ('le sei donne che in Arcadia venivano chiamate ninfe'): così, nell'utilizzare il termine nympha, Virgilio proprio ergo vocabulo, non epitheto poetico usus est ('ha usato il termine appropriato, non un epiteto poetico'). Per l'interpretazione virgiliana di Carmentis e altri problemi connessi alla sua identificazione, si può fare riferimento alla nota ad l. di Eden 1975,110 s. Cfr. anche Dion.Hal. 1,31,1; Strab. 5,230; [Aur.Vict.] Or.gent.Rom. 5,1; Isid. 1,4,1.

10. Merli 2000,285 ss. I Carmentalia sono celebrati, invero, in due date distinte: l'11 e il 15 gennaio. Carmenta condivide l'11 gennaio con un'altra divinità associata alle Camenae, Giuturna, ninfa delle sorgenti e - come sappiamo dall'Eneide - sorella di Turno, re dei Rutuli: in questo giorno (all'incirca a metà del III sec. a. C.) le era stato dedicato un tempio da Gaio Lutazio Catulo in Campo Martio (Serv. ad Aen. 12,139), in corrispondenza del tratto finale dell'Aqua Virgo (cfr. fast. 1,464 hic ubi Virginea Campus obitur Aqua, 'qui dove il Campo finisce nell'Acqua Vergine'), l'acquedotto che rifornisce d'acqua la Fontana di Trevi e quella di piazza Navona. Su monere e la sua connessione con le Camenae (e Moneta), si possono vedere Horsfall 2000 ad Aen. 7,645 e, in particolare, Hardie 2007,559 ss. e 580 s. (su Livio Andronico, Nevio e Virgilio).

11. Per sottolineare la pertinenza di questa immagine al contesto, Barchiesi 1994,186 s. richiama 1,499 s. iamque ratem doctae monitu Carmentis (...) egerat (scil. Evander), 'e già Evandro aveva condotto la nave seguendo il consiglio dell'esperta Carmenta', su cui cfr. ancora più oltre.

12. A tale proposito, uno dei referee mi suggerisce opportunamente che una tradizione riferita da Isidoro di Siviglia $(1,4,1$ e 5,39,11) attribuiva proprio a Carmenta l'introduzione dell'alfabeto latino presso i popoli dell'Italia.

13. Cfr. 505-8, dove in particolare l'espressione non sano ... pede (506) potrebbe alludere copertamente - come mi segnala sempre uno dei referee - ad una etimologia alternativa del nome di Carmenta (da carere mente), per cui cfr. Plut. vit. Rom. 21,1-3 e Quaest.Rom. 56.

14. In quanto protettrice delle nascite Carmenta era celebrata piuttosto il 15 gennaio. Nella circostanza, tuttavia, a testo non troviamo alcun segnale, né diretto né indiretto, dell'attribuzione a Carmenta del ruolo di ispiratrice silenziosa.

15. Cfr. Liv. 1,7,8: già venerabilis per aver introdotto la scrittura (cfr., qui sotto, la tradizione della sua discendenza paterna dal dio Hermes, 'inventore' della scrittura), Evandro lo era ancor più per il fatto di essere figlio di Carmenta, cui si attribuivano facoltà divine (venerabilior divinitate credita Carmentae matris).

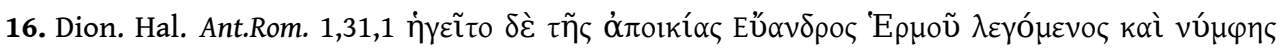

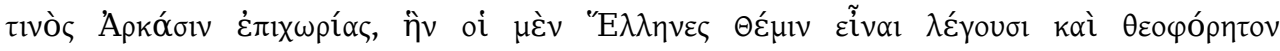

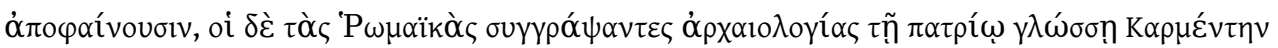

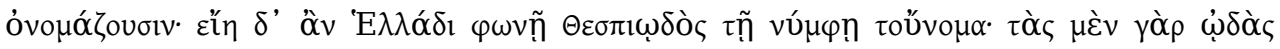

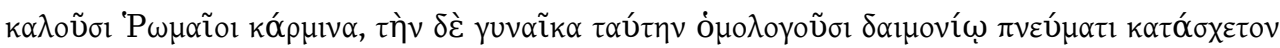

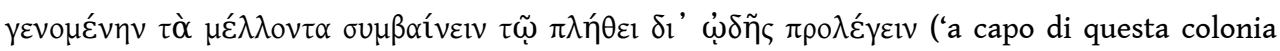
c'era Evandro, che - si dice - era figlio di Hermes e di una ninfa epicorica di Arcadia. I Greci la chiamano Themis e dicono che possedeva il dono dell'ispirazione profetica; gli autori romani, invece, la chiamano, nella loro lingua nazionale, Carmenta. Il nome di quest ninfa sarebbe in greco Thespiôdos; infatti i Romani chiamano carmina i canti oracolari, e concordano nel ritenere che questa donna, posseduta dall'ispirazione divina, era in grado di rivelare agli uomini il futuro col suo canto oracolare'). Questa tradizione, secondo una comune opinione, è attestata anche da Virgilio, ma nella frase vobis Mercurius pater est di Aen. 8,138 il pronome di II pers. pl. potrebbe anche riferirsi al popolo arcade nel suo complesso e non necessariamente solo ad Evandro e ai suoi discendenti.

17. Cfr. Serv.Dan. ad Aen. 8,130: al momento di spiegare perché Enea dice che Evandro ha dei legami di parentela con gli Atridi, Servio all'inizio attribuisce alla madre del re arcade il nome di Timandra e a suo padre quello di Echemus (quidam aiunt Thestii filias Ledam et Hypermestram fuisse, Ledae et Tyndarei filias Clytemestram Helenam et Timandram fuisse, quam duxit uxorem Echemus Arcas, 
cuius filius Euander). In seguito, egli aggiunge che, secondo altre fonti (alii ita tradunt), la madre di Evandro si chiamava Nicostrata ed era figlia di Hermes (Mercurius deinde est Nicostratae pater, quae fuit mater Euandri; cfr. Serv. ad Aen. 8,51 hic [scil. Euander] patrem suum occidit, suadente matre Nicostrata, quae etiam Carmentis dicta est, quia carminibus vaticinabatur e Serv. ad Aen. 8,336).

18. Cfr. la rievocazione dell'episodio da parte di Evandro in Virg. Aen. 8,335 s. matrisque egere tremenda / Carmentis nymphae monita (Barchiesi 1994,187).

19. Alcune note di Servio ci informano che Evandro avrebbe ucciso il padre e la madre (Serv. Aen. 8,51 e 333), mentre Dionigi di Alicarnasso (Ant.Rom. 1,31,2) parla più genericamente di una ribellione che aveva portato alla sua espulsione dall'Arcadia, insieme alla madre.

20. Cfr. tr. 5,4,17 s. nec fore perpetuam sperat sibi numinis iram, / conscius in culpa non scelus esse sua, 'e spera di non avere sempre il dio in collera con lui, conscio che nella sua colpa non c'è intento criminoso'; ma anche 1,5,44 deminui siqua numinis ira potest, 'se in qualche modo può essere mitigata la collera del dio'; 3,6,23 numinis ut laesi fiat mansuetior ira, 'affinché la collera del dio diventi più mite'; 4,8,50 plus tamen exitii numinis ira dedit, 'il più della mia rovina lo devo alla collera di un dio'; Pont. 2,1,47 s. cur ego posse negem minui mihi numinis iram, / cum videam mitis hostibus esse deos?, 'perché dovrei dire che l'ira del dio verso di me non può diminuire, quando vedo che gli dèi sono clementi con i nemici ?'; e anche tr. 1,10,41 s. a quibus adveniat Miletida sospes ad urbem, / offensi quo me detulit ira dei, 'da lì (= da Callatis) giunga poi sana e salva (scil. la nave) nella città milesia (= Tomi), dove l'ira dell'imperatore offeso mi ha destinato'; 5,10,51 s. quid loquor, ah, demens? ipsam quoque perdere vitam / Caesaris offenso numine, dignus eram!, 'che cosa sto dicendo, pazzo che non sono altro? Anche la vita ero degno di perdere, avendo offeso Augusto'; Pont. 1,10,42. Per numinis iram cfr. Ov. met. 2,659; 4,8; 6,313; 10,239. Ma ci sono anche altri termini di confronto significativi: fast. 1,484 crimen abesse (fast. 4,323; tr. 2,250 inque meo nullum carmine crimen erit, 'e nel mio poema non ci sarà nulla di criminoso'; la sequenza composta da Giasone, Cadmo e Tideo è la stessa che possiamo leggere in Pont. 1,3,75 ss.); $495 \mathrm{~s}$. nec fera tempestas toto tamen horret in anno / et tibi, crede mihi, tempora veris erunt, 'per quanto aspra, una tempesta non infuria per un anno intero: e anche per te, credimi, verrà la stagione di primavera' (cfr. tr. 2,141 s. e Pont. 4,4,1 s.).

21. Fantham 1992,166-70. La revisione del poema, e di questo episodio in particolare, nell'ultima fase della vita e dell'esilio di Ovidio sembrano acquisizioni sicure, come dimostrano anche alcuni indizi interni: come il titolo di Iulia Augusta $(1,536)$ attribuito a Livia, che lo ricevette nel 14 d.C. alla morte di Augusto (Green 2004,236 s.; Pasco-Pranger 2006,191).

22. Green 2004,221.

23. Varr. Ant. fr. 103 Card. altera Postverta cognominata est, Prorsa altera, a recti perversique partus et potestate et nomine, 'una è chiamata Postverta, l'altra Prorsa, nomi collegati al fatto che proteggono rispettivamente il parto che avviene in senso corretto e quello in senso contrario'; Tert. nat. $2,11,6$.

24. 1,633-6 Porrima placatur Postvertaque, sive sorores, / sive fugae comites, Maenali diva, tuae; / altera quod porro fuerat cecinisse putatur, / altera venturum postmodo quicquid erat, 'si placano Porrima e Postverta, sia che fossero tue sorelle, sia che fossero tue compagne di fuga, o signora divina del Menalo; l'una si crede che cantasse ciò che era accaduto prima, l'altra ciò che sarebbe avvenuto in seguito', dove si può osservare l'impiego catacrestico di porro nel significato temporale di 'davanti/prima', equivalente di antea (cfr. Serv. ad Aen. 8,336 alii huius comites Porrimam et Postvertam tradunt, quia vatibus et praeterita et futura sunt nota, 'altri tramandano che sue compagne furono Porrima e Postverta, poiché ai profeti è noto sia il passato che il futuro'). Le due paredre di Carmenta finiscono così per coincidere con Antevorta e Postvorta, paredre di Giano (Macr. $1,7,20)$.

25. Dopo la vittoria - la cui rappresentazione risente evidentemente dello scenario della gigantomachia (e dove Caco assomiglia a Tifeo) - Ercole celebra di persona sia il sacrificio di ringraziamento in onore di Giove (579 s.), che l'atto di fondazione dell'Ara Maxima nel Forum 
Boarium (581 s.). Alla matrice sacerdotale di questa caratterizzazione dell'eroe contribuisce anche l'unica frase che egli pronuncia: 561 accipio revocamen, 'accolgo il richiamo' (sull'importanza del tema del sacerdozio, cfr. anche 462 Arcadiae sacrum pontificale deae, 'il rito pontificale sacro alla dea di Arcadia').

26. Che si manifesta al poeta per svolgere il ruolo di informatore $(3,171 \mathrm{ss}$.$) , deponendo solo in$ parte i suoi attributi bellici (l'elmo, ma non l'asta).

27. Merli 2000,117 ss.

28. Sulla funzione di Egeria come occasionale Musa ispiratrice del poeta, cfr. Buchheit 1993,81 s.

29. Ancora prima, Marte stesso aveva (forse) 'suggerito' al poeta (3,167 occulti monitus) la storia della nascita di Romolo, spiegando con orgoglio che quest'ultimo aveva poi voluto dedicare a lui, Marte, suo padre, il primo mese dell'anno.

30. Secondo la tradizione più antica Ares-Mars è nato da Zeus-Jupiter ed Hera-Giunone, ma accanto a questa - i Fasti presentano altrove una versione ben diversa della storia, attraverso la testimonianza di una informatrice divina maliziosa e simpatica: Flora (5,229 ss.; cfr. Fucecchi 2004,32 ss.).

31. Sull'episodio che segue e il suo significato estetico ed ideologico nell'economia del poema, si vedano soprattutto Stok 2004,69 ss. e Labate 2010,207-10.

32. Merli 2013,7.

33. Sulla relazione ideale tra Numa, re colto ed ispirato, e l'erudito autore dei Fasti, cfr. Hinds 1992 e Pasco-Pranger 2006,90 s.

34. Ov. Pont. 1,9,23 placabilis ira deorum est, 'la collera degli dèi può essere placata'; cfr. anche tr. $4,9,14$ saepe Iovis telo quercus adusta viret; $1,3,11$ e Pont. 3,6,17 s. fulminis adflatos interdum vivere telis / vidimus et refici non prohibente Iove, 'abbiamo visto che chi è stato colpito dai dardi del fulmine talora vive e si riprende senza che Giove lo proibisca'. Per l'impiego di placabilis in relazione alla possibilità di neutralizzare l'effetto della folgore, cfr. anche Sen. nat. 2,43,1 fulmen quod ... Iuppiter mittit placabile est?, 'si può stornare il fulmine che scaglia Giove ?'.

35. Basti pensare a un informatore molto 'sui generis' come il dio Marte.

36. Queste allusioni alla situazione del poeta esule, così come ci vengono indirettamente veicolate dalle parole di Carmenta ed Egeria, possono risuonare perfino più chiare di molti riferimenti rintracciabili nelle elegie dell'esilio.

\section{RIASSUNTI}

Questo contributo si occupa in particolare di due personaggi femminili di altrettanti episodi dei Fasti: Carmenta - la madre del re Evandro, esule dall'Arcadia stabilitosi nel Lazio prima dell'arrivo di Enea -, ed Egeria, la sposa-consigliera del re Numa. Anche a queste figure 'divine', incluse nel novero delle Camenae o comunque ad esse a vario titolo collegate, il poema ovidiano sul calendario attribuisce il ruolo di informatrici del poeta-eziologo : ma si tratta di informatrici molto particolari. A differenza dei numerosi interlocutori divini le cui epifanie animano scene di vero e proprio dialogo, Carmenta ed Egeria svolgono infatti la loro funzione 'silenziosamente', ispirando il canto del narratore in modo discreto e ottenendo da lui una celebrazione delle loro prerogative e dei loro meriti. In cambio, quando le due ispiratrici divine prendono direttamente la parola per rivolgersi ad altri personaggi dei rispettivi racconti, il narratore principale fa in modo che esse gli restituiscano, per così dire, il favore. Le due lunghe rheseis di Carmenta, così 
come alcune allocuzioni di Egeria (l'incoraggiamento e il consiglio a Numa), contengono espressioni consolatorie che richiamano la situazione esistenziale (l'esilio, in particolare) vissuta da Ovidio in quanto autore empirico.

INDICE

Mots-clés : elegia eziologica, voce, punto di vista narrativo, storia romana, esilio

\section{AUTORE}

MARCO FUCECCHI

Udine 